\title{
Dosage de I'ATP bactérien du lait cru par l'appareil BactoFoss
}

\author{
D Rongvaux-Gaïda, C Piton-Malleret, A Dasen
}

\author{
INRA, station de recherches en technologie et analyses laitières, \\ BP 89, 39801 Poligny Cedex, France
}

(Reçu le 10 avril 1991; accepté le 14 juin 1991)

\begin{abstract}
Résumé - Le BactoFoss (Foss Electric) est une technique automatique de dosage de l'ATP bactérien qui a été évaluée comme méthode rapide d'appréciation de la qualité bactériologique du lait cru. Dans une première étape, nous avons vérifié l'effet non significatif de la conservation des échantillons de lait à $0^{\circ} \mathrm{C}$ avant analyse, sur le signal mesuré par le BactoFoss, et nous avons montré que l'homogénéisateur à billes (Foss Electric) couplé au BactoFoss possède un pouvoir de dispersion des amas microbiens équivalent à l'Ultra-turrax. Grâce à des dilutions successives de 5 échantillons de lait cru dans du lait UHT, nous avons mis en évidence que la réponse du BactoFoss est linéaire sur une gamme de concentration de $10^{3}$ à $10^{7} \mathrm{UFC} / \mathrm{ml}$, mais que la quantité moyenne d'ATP par cellule varie d'un échantillon à l'autre : les droites de régression obtenues entre Référence et BactoFoss pour les différents échantillons sont parallèles et non confondues. Dans une seconde étape, nous avons établi les parametres de répétabilité et de justesse du BactoFoss, sur 120 échantillons de lait prélevés dans les citernes de ramassage de 4 grandes entreprises laitières et sur 118 échantillons prélevés dans les tanks de différents producteurs. L'écart type de répétabilité du BactoFoss est en moyenne de $0,108 \mathrm{log} \mathrm{UFC} / \mathrm{ml}$, ce qui correspond à un écart type relatif géométrique de $28 \%$. Sur les 238 échantillons couvrant une gamme de concentration de $10^{3}$ à $10^{7} \mathrm{UFC} / \mathrm{ml}$, l'écart type résiduel de la régression BactoFoss sur Référence est de 0,495 log UFC/ml. La précision d'estimation du BactoFoss est significativement meilleure pour les échantillons de lait de citernes $\left(s_{y, x}=0,420\right.$ $\log \mathrm{UFC} / \mathrm{ml}$ ) que pour les échantillons de lait de tanks $\left(s_{y, x}=0,556 \log \mathrm{UFC} / \mathrm{ml}\right)$. De même, la corrélation entre la référence et le BactoFoss est meilleure lorsqu'on compare les résultats du BactoFoss à ceux de numérations obtenus après agitation standard $\left(s_{y, x}=0,420 \log \mathrm{UFC} / \mathrm{ml}\right)$ plutôt qu'après passage à l'Ultra-turrax $\left(s_{y, x}=0,499 \log\right.$ UFC/ml).
\end{abstract}

BactoFoss / ATP / lait cru / flore totale / justesse / répétabilité / agitation

Summary - Determination of bacterial ATP level in raw milk with BactoFoss instrument. The BactoFoss (Foss Electric) is an automatic technique to estimate the bacterial ATP level, which has been tested as a rapid method for the assessment of the bacteriological quality of raw milk. In the first part of this study, we have checked the non-significant effect of the storage of the samples at $0{ }^{\circ} \mathrm{C}$ before analysis on the BactoFoss signal, and we have shown that the homogenizer (Foss Elec- 
tric) used with BactoFoss is as efficient as the Ultra-turrax in breaking up the microbial aggregates. With successive dilutions of 5 raw milk samples in UHT milk, we have shown that the BactoFoss response is linear from $10^{3}$ to $10^{7} \mathrm{CFU} / \mathrm{ml}$, but that the average level of ATP per cell varies between samples: the regression lines obtained between the reference and BactoFoss signal for the different samples are parallel. In the second part of the study, we have established the repeatability and the accuracy of the BactoFoss on 120 dairy plant milk samples taken from 4 plants and 118 farm milk samples. The repeatability standard deviation of BactoFoss averages $0.108 \mathrm{log}$ CFU/ml, corresponding to a geometric relative standard deviation of $28 \%$. On the 238 samples ranging from $10^{3}$ $10^{7} \mathrm{CFU} / \mathrm{ml}$, the residual standard deviation of the regression reference on BactoFoss signal is $0.495 \mathrm{log}$ CFU/ml. The accuracy of the BactoFoss is significantly better for the dairy plant samples $\left(s_{y, x}=0.420 \log\right.$ CFU/ml) than for the farm milk samples $\left(s_{y, x}=0.556 \log\right.$ CFU/ml). The correlation between the reference and the BactoFoss is better when the BactoFoss signals are compared with the numbers of CFU/ml obtained after a standard shaking of samples $\left(s_{y_{1} x}=0.420 \mathrm{log}\right.$ CFU/ml) than after an Ultra-turrax shaking $\left(s_{y, x}=0.499 \log\right.$ CFU/ml).

BactoFoss / ATP / raw milk / total flora / accuracy / repeatability / shaking method

\section{INTRODUCTION}

L'ATP représente un marqueur biotique de choix, car sa présence est indispensable aux processus de transfert d'énergie dans toutes les cellules. Sa concentration résulte d'un équilibre permanent entre les mécanismes de production et d'utilisation, et il disparaît rapidement après la mort cellulaire. Toutefois, on ne connaît pas avec précision l'importance des variations de sa concentration au sein de la cellule en fonction de son état physiologique et/ou des différentes espèces microbiennes.

Bien que le dosage de I'ATP par bioluminescence soit une méthode très sensible, simple, rapide et de plus spécifique des cellules vivantes, cette technique n'a pas encore sa place comme méthode rapide d'appréciation de la qualité bactériologique du lait cru en usine, en raison de ses médiocres performances analytiques. En effet, le dosage précis de l'ATP bactérien du lait est entravé par un bruit de fond important lié à la présence de cellules somatiques et de micelles de caséines, contenant toutes deux de I'ATP (Bossuyt, 1978; Theron et al, 1986). L'élimination complète de ce bruit de fond est difficile, voire même impossible sans altérer l'ATP bactérien (Theron et al, 1986).

Pour répondre aux besoins de l'industrie alimentaire, une nouvelle technique de dosage, l'ATP-F test (Lumac), basée sur une séparation des différentes sources d'ATP par filtration, et une concentration des bactéries sur le filtre a récemment été présentée par Waes et al (1989). Cette méthode possède une précision d'estimation et un seuil de détermination nettement plus satisfaisants que les techniques rapides de dosage de I'ATP du lait cru comme l'ATP platform test (Bossuyt, 1982) ou le $\triangle$ ATP test (Langeveld et Van der Waals, 1988; Rongvaux-Gaïda et Piton, 1991). Dans un même temps, Foss Electric a mis au point un appareil entièrement automatique de dosage de l'ATP (BactoFoss), basé sur le même principe.

Jusqu'à présent, peu de travaux ont été consacrés à cet appareil (Eriksen et Olsen, 1988; Olsen et Eriksen, 1989). De plus les résultats disponibles sont tous issus de prototypes de laboratoire. Dans le cadre de cette étude, nous avons donc cherché à établir les paramètres de répétabilité et 
de justesse du BactoFoss, dans sa version commercialisée, pour l'estimation du niveau de pollution microbienne d'échantillons de lait cru prélevés dans les citernes de ramassage de plusieurs entreprises ou chez différents producteurs. Nous avons dans un premier temps vérifié différents points du protocole et de la technique, c'est-à-dire, l'effet de la conservation des échantillons à $0{ }^{\circ} \mathrm{C}$ sur le signal mesuré par le BactoFoss, la linéarité de réponse de l'appareil sur une gamme de concentration comprise entre $10^{2}$ et $10^{8} \mathrm{UFC} / \mathrm{ml}$, et l'efficacité de dispersion des amas bactériens par l'homogénéisateur associé au dosage.

\section{MATÉRIEL ET MÉTHODES}

\section{Origine des échantillons}

Pour étudier l'effet du stockage à $0^{\circ} \mathrm{C}$ sur le signal bactérien, 2 échantillons de lait cru ont été prélevés aussitôt après la traite. À l'arrivée au laboratoire, ils ont été divisés en 10 à 15 fractions de $50 \mathrm{ml}$. L'une d'elles a été analysée avant refroidissement, tandis que les autres ont été stockées à $0^{\circ} \mathrm{C}$ dans la glace fondante pendant $3 \mathrm{j}$. Ces fractions ont été analysées à diverses reprises au cours de ces $3 \mathrm{j}$, de façon à calculer la reproductibilité du signal fourni par I'ATP bactérien au cours du temps.

Pour tester la justesse et la linéarité de réponse du BactoFoss sur une gamme de concentration bactérienne très étendue, 5 échantillons de lait cru ont été prélevés chez différents producteurs; 4 d'entre eux ont été maturés à $14{ }^{\circ} \mathrm{C}$ pendant un temps compris entre 15 et $48 \mathrm{~h}$, de façon à atteindre des niveaux de pollution microbienne élevés. Tous ces échantillons ont été bloqués à $0^{\circ} \mathrm{C}$ dans la glace fondante pendant au moins $3 \mathrm{~h}$ avant analyse. Ils ont ensuite été soumis à une série de dilutions successives dans du lait UHT demi-écrémé, de façon à couvrir une gamme de concentration variant entre $10^{2}$ et $10^{8} \mathrm{UFC} / \mathrm{ml}$.
Pour étudier les caractères de répétabilité et de justesse du BactoFoss, 120 échantillons de lait cru ont été prélevés dans les citernes de ramassage de 4 entreprises laitières françaises, dès leur arrivée au quai de réception; ils ont été conservés à $0^{\circ} \mathrm{C}$ au plus $24 \mathrm{~h}$ avant analyse. D'autre part, 118 échantillons de lait cru ont également été prélevés chez des producteurs du département du Jura; une partie d'entre eux ont été maturés à $14{ }^{\circ} \mathrm{C}$ afin d'augmenter leur niveau de pollution microbienne. D'une façon générale, tous ces échantillons ont été bloqués à $0{ }^{\circ} \mathrm{C}$ dans la glace fondante au minimum $3 \mathrm{~h}$ avant analyse, pour travailler dans des conditions opératoires standardisées.

\section{Numérations bactériennes}

Avant ensemencement, tous les échantillons ont subi une agitation standard qui consiste en 25 mouvements aller-retour d'une amplitude de 25$30 \mathrm{~cm}$ effectués en 7-10 s (Anonyme, 1978).

Les échantillons de lait ont ensuite été soumis au dénombrement de la flore aérobie mésophile sur milieu Plate Count Agar (Difco) après 3 j d'incubation à $30^{\circ} \mathrm{C}$.

Les ensemencements ont été réalisés à la surface de la gélose à l'aide de l'appareil Spiral (Gilchrist et al, 1973). Nous avons choisi cette méthode, car elle est maintenant reconnue comme technique de référence (Anonyme, $1985 b$ et 1987).

Les 120 échantillons de lait de citernes ont également subi une agitation violente pendant $30 \mathrm{~s}$ à l'aide d'un homogénéisateur à turbine (Ultra-turrax, type 18/10) tournant à $20000 \mathrm{tr} /$ min. Ce mode d'agitation est actuellement reconnu comme celui qui assure la meilleure dispersion des amas microbiens (Richard, 1980; Richard et al, 1981). Après agitation, ces 120 échantillons ont alors été soumis au dénombrement de la flore aérobie mésophile sur milieu PCA après $3 \mathrm{j}$ d'incubation à $30^{\circ} \mathrm{C}$ et de la flore psychrotrophe sur le même milieu, après $10 \mathrm{j}$ d'incubation à $7^{\circ} \mathrm{C}$.

Enfin, 60 échantillons pris au hasard parmi les 120 prélevés dans les citernes de ramassage ont été également soumis au dénombrement de la flore totale, après passage à l'homogénéisateur à billes Foss Electric. 
Les ensemencements ont été effectués en double pour chaque échantillon, afin d'obtenir une meilleure précision des résultats. Les résultats sont exprimés en unités formant colonies (UFC) par ml de lait.

\section{BactoFoss}

Le principe du BactoFoss repose sur une séparation physique de I'ATP des cellules somatiques et de l'ATP bactérien et sur une concentration des bactéries par filtration.

Après agitation, une fraction de l'échantillon $(25 \mathrm{ml})$ est passée dans un homogénéisateur à billes (Foss Electric) de façon à assurer une dispersion des amas microbiens. L'appareil prélève ensuite automatiquement $70 \mu \mathrm{l}$ de l'échantillon agité et le dépose sur un papier filtre au fond d'un puits thermostaté $\left(50^{\circ} \mathrm{C}\right)$. Par une série de traitements, lavages et filtrations successifs, I'ATP d'origine somatique est extrait et éliminé; les microorganismes se retrouvent alors isolés sur le papier filtre. Celui-ci est entraîné dans la chambre de mesure où l'ATP bactérien est extrait et dosé. La quantité d'ATP libéré est déterminée par la mesure de la lumière émise après addition du réactif luciférineluciférase. Les résultats s'affichent sur écran digital et sont transmis directement sur imprimante.

Un cycle complet d'analyse d'un échantillon de lait cru dure $3 \mathrm{~min}$. Les résultats sont exprimés en nombre de cellules/ml de lait.

\section{Calculs statistiques}

La répétabilité et la justesse des dosages d'ATP ont été calculées conformément aux recommandations de la norme FIL 128 (Anonyme, 1985a). Les calculs statistiques ont été effectués après transformation logarithmique en base 10 des résultats (UFC/ml et nombre de cellules/ml). Cette transformation est destinée à normaliser la distribution.

Tous les modes de calcul statistique ont été largement détaillés dans un précédent article (Piton et Rongvaux-Gaïda, 1990); aussi, nous n'en rappelons ici que les points les plus importants pour la compréhension des résultats.

\section{Répétabilité}

L'écart type de répétabilité des dosages d'ATP $(s$, en nombre de cellules $/ \mathrm{ml}$ ) a été établi à partir de 2 mesures effectuées sur chaque échantillon (Anonyme, 1985a). Pour pouvoir comparer la répétabilité de la méthode à celles des autres techniques, nous l'avons également exprimée en $\log \mathrm{UFC} / \mathrm{ml}\left(s_{r}^{*}\right)$ par l'intermédiaire de la droite de calibrage.

Enfin, nous avons calculé l'écart type relatif géométrique de répétabilité, ou GRSD, qui exprime la variabilité des résultats au-dessus de la moyenne géométrique en données non transformées (Piton et Grappin, 1991).

\section{Justesse}

La justesse représente l'étroitesse de l'accord entre la valeur de la grandeur à mesurer et le résultat moyen qui serait obtenu en appliquant la technique expérimentale un grand nombre de fois (Anonyme, 1985a).

Dans le cadre de cette étude, l'équation de calibrage $(y=b x+a)$ a été établie conformément à la norme FIL. Le logarithme décimal du nombre d'UFC/ml (valeur vraie) est considéré comme variable dépendante et le log du nombre de cellules déterminé par le BactoFoss (moyenne de 2 déterminations) comme variable indépendante.

La précision d'estimation de la technique expérimentale est donnée par les limites de l'intervalle de confiance à l'intérieur duquel se situe, avec une probabilité de $95 \%$, la vraie valeur donnée par la méthode de référence. Si $y$ est le nombre d'UFC/ml calculé à partir de la droite de calibrage, ces limites sont, en valeur logarithmique, $\log y \pm 1,96 \times s_{y, x}$, où $s_{y, x}$ est l'écart type résiduel des écarts à la droite de régression. En données non transformées, ces limites sont $y / 10^{1,96 \times s_{y \times x}}$ pour la limite inférieure et $\mathrm{y} \times 10^{1,96 \times s_{y, x}}$ pour la limite supérieure. 


\section{RÉSULTATS}

\section{Effet du stockage des échantillons de lait à $0^{\circ} \mathrm{C}$}

Le stockage à $0^{\circ} \mathrm{C}$ avant dosage est imposé par le délai écoulé entre la récolte des échantillons et leur analyse, en raison de leur transport et de l'organisation du travail au laboratoire. Comme la température influence le métabolisme cellulaire, et par conséquent, la teneur en ATP, nous avons voulu vérifier l'existence d'un impact éventuel du stockage à $0{ }^{\circ} \mathrm{C}$ sur le dosage de I'ATP. La figure 1 présente, pour 2 échantillons de lait, l'évolution du signal donné par I'ATP bactérien en fonction de la durée de stockage à $0^{\circ} \mathrm{C}$. La première mesure effectuée au temps 0 , correspond à l'échantillon non refroidi $\left(20-25^{\circ} \mathrm{C}\right)$, analysé aussitôt après la traite, tandis que les mesures suivantes ont été réalisées sur les échantillons refroidis et maintenus à $0{ }^{\circ} \mathrm{C}$ jusqu'au moment de l'analyse. On n'observe pas de changement net lié au refroidissement du lait, ni même d'évolution du signal au cours des $3 \mathrm{j}$ de stockage. En effet, les logarithmes des nombres de cellules/ml oscillent autour des moyennes respectivement de 4,72 et de 4,40 pour les échantillons 1 et 2 , et les écarts types de reproductibilité $\left(s_{R}\right)$ des nombres de cellules mesurés au cours des 3 j d'analyse sont de 0,11 log cellules $/ \mathrm{ml}$ pour l'échantillon 1 et 0,09 log cellules $/ \mathrm{ml}$ pour l'échantillon 2.

\section{Importance de la dispersion des amas microbiens par l'homogénéisateur Foss Electric}

Le BactoFoss utilisant un volume d'échantillon de $70 \mu \mathrm{l}$ pour doser l'ATP bactérien du lait cru, un homogénéisateur lui est cou-

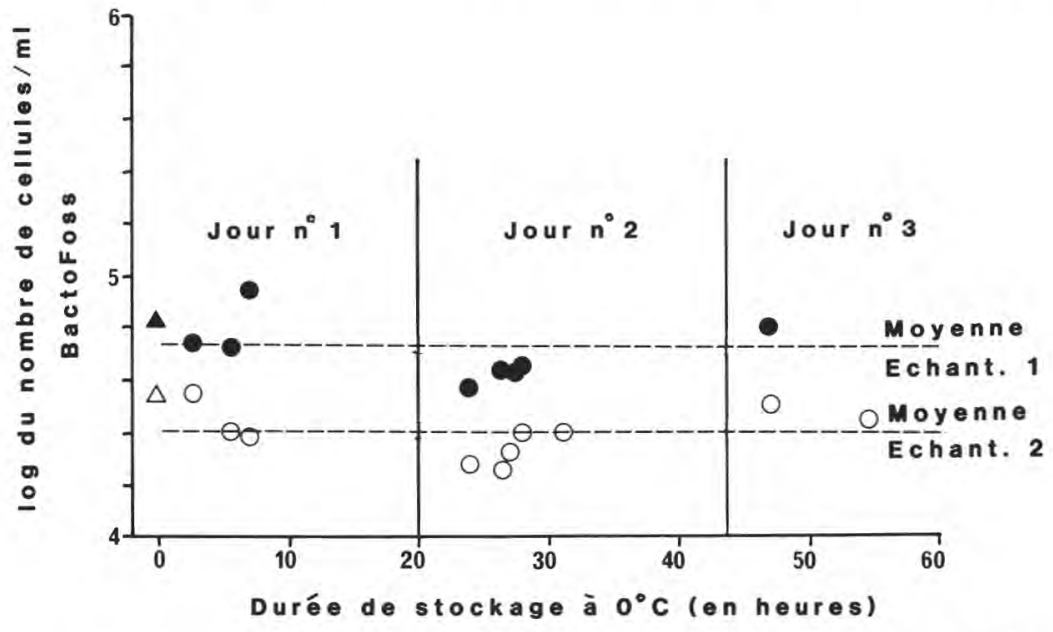

Fig 1. Effet du stockage des échantillons de lait à $0^{\circ} \mathrm{C}$ sur le signal mesuré par le BactoFoss. $\Delta$ et $\boldsymbol{\Delta}$ : échantillons 1 et 2 non refroidis, et analysés aussitôt après la traite; $O$ et : échantillons 1 et 2 analysés après refroidissement à $0^{\circ} \mathrm{C} ;---$ : moyenne.

Effect of keeping the milk samples at $0^{\circ} \mathrm{C}$ on the signal measured by BactoFoss. $\Delta$ et $\mathbf{\Delta}$ : samples 1 and 2 not cooled and analysed immediately after milking; $O$ et $:$ samples 1 and 2 analysed after cooling at $0{ }^{\circ} \mathrm{C} ;---$ : mean. 
plé pour s'assurer que le microvolume analysé soit représentatif de l'ensemble de l'échantillon. Nous avons cherché à évaluer les conséquences de cette agitation intense des échantillons sur la dispersion des amas microbiens en comparant les résultats de numération obtenus après passage à l'homogénéisateur à ceux obtenus par agitation standard d'une part, et par agitation à l'Ultra-turrax d'autre part.

Par rapport à l'agitation standard, l'homogénéisateur entraîne une augmentation significative des résultats de numération (fig 2A). Pour 59 échantillons, les moyennes sont en effet respectivement de 5,214 et 4,885 en log UFC/ml, ce qui correspond à une multiplication des nombres d'UFC/ml par un facteur 2,13 après passage à l'homogénéisateur. Par ailleurs, la pente de la droite de calibrage est significativement inférieure à 1,000 au seuil de $5 \%$, indiquant que l'écart entre modes d'agitation varie en fonction du niveau de contamination du lait : cet écart est très faible pour les échantillons peu contaminés et augmente avec le niveau de pollution du lait.

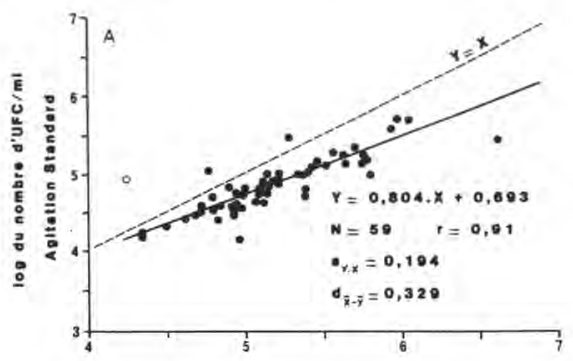

La relation obtenue entre les nombres d'UFC/ml déterminés après agitation par turbine ou par homogénéisateur à billes est plus étroite (fig 2B). En effet, la précision d'estimation passe de $\pm 1,96 \times 0,194$, c'est-à-dire $\pm 0,380 \log$ UFC/ml à $\pm 1,96 \times$ 0,108 , c'est-à-dire $\pm 0,212 \log U F C / m l$. D'autre part, sur 57 échantillons analysés, les moyennes sont respectivement de 5,20 pour l'homogénéisateur et de 5,22 pour la turbine et ne sont pas significativement différentes au seuil de $5 \%$. Cependant, la pente de la droite reste significativement inférieure à 1000 au seuil de $5 \%$.

\section{Répétabilité}

Le tableau I montre l'évolution de l'écart type de répétabilité du BactoFoss en fonction du niveau de contamination des échantillons. Bien que cet écart type varie de façon significative en fonction de la classe, on ne peut mettre en évidence aucune évolution nette de cet écart type avec le niveau de pollution microbienne. En moyenne, sur les 238 échantillons de lait

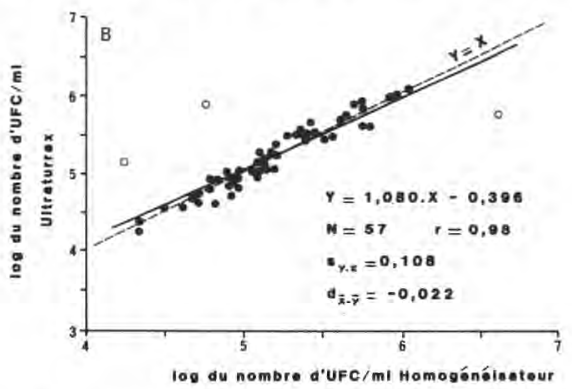

Fig 2. Influence de l'homogénéisateur Foss Electric sur les résultats de numération de la flore totale du lait cru. A. Comparaison avec une agitation standard; B. Comparaison avec une agitation Ultraturrax. $s_{y, x}$ : écart type résiduel de la régression en log UFC/ml; $N$ : nombre d'échantillons; $r$ : coefficient de corrélation; $d_{\bar{x}-\bar{y}}$ : différence entre les moyennes en log UFC/ml. Les droites de calibrage ont été calculées après élimination des échantillons (o) qui s'écartaient de plus en plus de 3 écarts types résiduels de ces droites.

Influence of the Foss Electric homogenizer shaking method on the results of enumeration of total flora in raw milk. A. Comparison with standard shaking; $\boldsymbol{B}$. Comparison with Ultra-turrax shaking; $s_{y, x}$ : re$s$-idual standard deviation of the regression in $\log$ UFC/ml; $N$ : number of samples; $r$ : correlation coefficient; $d_{\bar{x}-\bar{y}}$ : différence between the means in log CFU/ml. 0 : samples omitted for the calculation of the regression lines. 
Tableau I. Influence du niveau de contamination des échantillons sur la répétabilité du BactoFoss. $\mathrm{N}$ : nombre d'échantillons; $\mathrm{s}_{\mathrm{r}}{ }^{*}$ : écart type de répétabilité en $\log U F C / m l$, calculé à partir de la pente de la droite de calibrage; GRSD : écart type relatif géométrique de répétabilité en \% $\mathrm{UFC} / \mathrm{ml}$.

Influence of the contamination level of the milk samples on the repeatability of BactoFoss. N: number of samples; $s_{r}{ }^{*}$ : repeatability standard deviation in log CFU/ml; GRSD : geometric relative standard deviation of repeatability in \% CFU/ $\mathrm{ml}$.

\begin{tabular}{lrrr}
\hline $\begin{array}{l}\text { Classes } \\
\text { en UFC } / \mathrm{ml}\end{array}$ & $N$ & $S_{r}{ }^{*}$ & $\begin{array}{l}\text { GRSD } \\
(\%)\end{array}$ \\
\hline$<5 \times 10^{4}$ & 86 & 0,111 & 29,12 \\
$5 \times 10^{4}-1 \times 10^{5}$ & 54 & 0,067 & 16,68 \\
$1 \times 10^{5}-1 \times 10^{6}$ & 64 & 0,139 & 37,72 \\
$>1 \times 10^{6}$ & 34 & 0,071 & 17,76 \\
$5 \times 10^{3}-7,6 \times 10^{7}$ & 238 & 0,108 & 28,23 \\
\hline
\end{tabular}

analysés, l'écart type de répétabilité est de $0,108 \log \mathrm{UFC} / \mathrm{ml}$, ce qui correspond à un écart type relatif géométrique (GRSD) de $28 \%$.

\section{Justesse}

Outre la classique comparaison des résultats entre référence et méthode indirecte effectuée sur une grande population d'échantillons, la justesse a également été abordée dans cette étude, à l'aide de dilutions successives, dans du lait UHT, d'échantillons de lait présentant des écarts plus ou moins grands par rapport à la référence.

La figure 3 présente les relations obtenues entre les nombres d'UFC/ml et les nombres de cellules/ml mesurés par le BactoFoss, pour les dilutions successives de 5 échantillons de lait cru; la présenta- tion des résultats a été dissociée en 3 figures, compte tenu des 3 types de réponse observés.

Pour les échantillons 1 et 2 , les dilutions ont couvert la gamme de concentration habituellement rencontrée pour les échantillons de producteurs, soit entre $10^{4}$ et $10^{6}$ $\mathrm{UFC} / \mathrm{ml}$ (fig $3 \mathrm{~A}$ ). La réponse du BactoFoss est linéaire dans cette gamme de concentration. Cependant les résultats sont significativement supérieurs à la référence au seuil de $5 \%$ (en moyenne de $30 \%$ ).

Pour l'échantillon 4 (fig 3B), les résultats couvrent une gamme de concentration variant de $10^{2}$ à $10^{5} \mathrm{UFC} / \mathrm{ml}$. Entre $10^{3}$ et $10^{5} \mathrm{UFC} / \mathrm{ml}$, la réponse du BactoFoss est

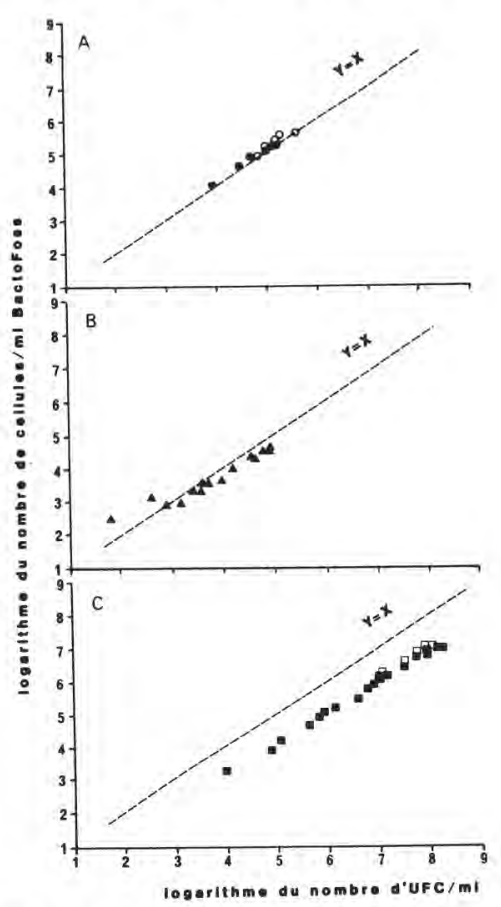

Fig 3. Justesse et linéarité de réponse du BactoFoss. A. Échantillons 1 (1) et 2 (O); B. Échan-

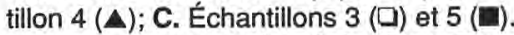
Accuracy and linearity of the BactoFoss signal. A. Samples 1 ( ) and $2(0)$; B. Sample 4 (4); C. Samples 3 (口) and 5 (घ). 
linéaire, mais contrairement aux échantillons précédents, les résultats du BactoFoss sont sous-estimés de façon significative par rapport à la technique Spiral : en moyenne, sur 12 échantillons, le BactoFoss donne 3,88 log cellules/ml, contre $4,14 \mathrm{log}$ UFC/ml pour le Spiral, soit une sous-estimation des nombres d'environ $45 \%$. À l'inverse, au-dessous de $10^{3}$ $\mathrm{UFC} / \mathrm{ml}$, il existe une rupture de pente qui indique une nette surestimation des nombres d'UFC/ml par le BactoFoss.

Enfin, la figure $3 \mathrm{C}$ présente les résultats obtenus pour les échantillons 3 et 5 . Tous 2 avaient subi une longue maturation à $14^{\circ} \mathrm{C}$ de façon à atteindre de très hauts niveaux de contamination (supérieurs à $10^{8} \mathrm{UFC} / \mathrm{ml}$ ). Au-delà de $10^{7}$ cellules $/ \mathrm{ml}$, le BactoFoss ne donne plus de résultats précis, mais une réponse de type "supérieur à". Au-dessous de cette valeur, la réponse du BactoFoss est linéaire: le coefficient de corrélation linéaire entre les logarithmes des nombres d'UFC/ml et des nombres de cellules/ml (BactoFoss) est de 0,99 . Par contre, le BactoFoss a très nettement sous-évalué le nombre de bactéries présentes dans ces 2 échantillons, et ce, sur toute la gamme de concentration. Sur les 15 dilutions de l'échantillon $n^{\circ} 5$, au-dessous de $10^{7}$ cellules/ml, les moyennes obtenues sont respectivement de 6,30 log UFC/ml pour le Spiral et de 5,35 log cellules/ml pour le BactoFoss, ce qui correspond à une sousestimation par le BactoFoss d'environ une unité logarithmique. D'autre part, la pente de la droite de régression est égale à 0,94 , et elle est significativement inférieure à 1,000 au seuil de $5 \%$.

La figure 4 présente la relation obtenue entre les logarithmes des nombres de cellules BactoFoss et des nombres d'UFC/ml après agitation standard, pour les échantillons de lait prélevés dans les citernes de ramassage (fig $4 A$ ) ou chez les produc- teurs (fig 4B). Pour les laits de citernes, la pente et l'ordonnée à l'origine de la droite de calibrage ne sont pas significativement différentes, respectivement de 1,000 et de 0,000 au seuil de $5 \%$, ce qui indique que le BactoFoss est un appareil correctement calibré. À l'inverse, avec un écart type $s_{b}$ de 0,079 , la pente de la droite de calibrage obtenue pour les échantillons de lait préle-

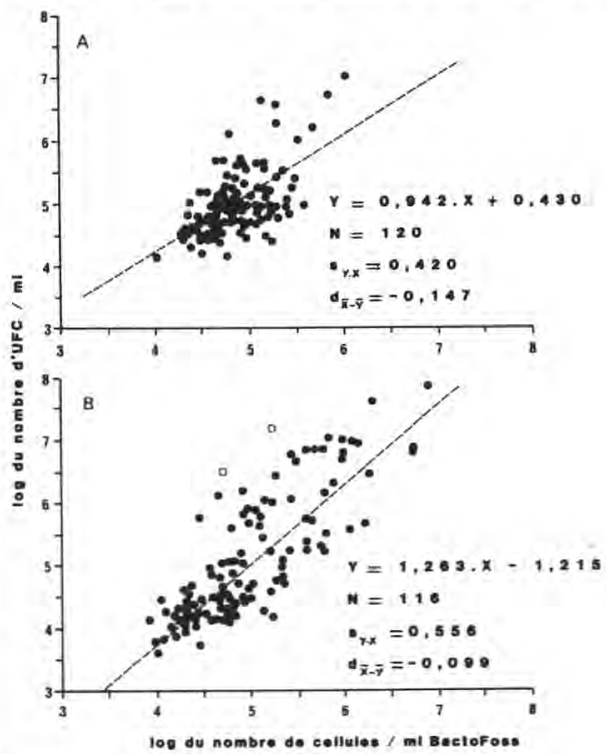

Fig 4. Relation entre les logarithmes des nombres d'UFC/ml (Spiral) et des nombres de cellules/ml (BactoFoss). A. Échantillons de citernes de ramassage; B. Échantillons de producteurs. $s_{y, x}$ : écart type résiduel de la régression en log UFC/ml; $N$ : nombre d'échantillons; $d_{\bar{x}-\bar{y}}$ : différence entre les moyennes en log UFC/ $\mathrm{ml}$. Les droites de calibrage ont été calculées après élimination des échantillons (o) qui s'écartaient de plus de 3 écarts types résiduels de ces droites.

Relationships between the logarithms of the numbers of CFU/ml (Spiral) and the numbers of cells/ml (BactoFoss). A. Dairy plant samples; B. Farm milk samples. $s_{y, x}$ : residual standard deviation of the regression in $\log \mathrm{CFU} / \mathrm{ml} ; \mathrm{N}:$ number of samples; $d_{\bar{x}-\bar{y}}:$ difference between the means in log CFU/mI. O: samples omitted for calculation of the regression lines. 
vés chez les producteurs est supérieure à 1,000 au seuil de $5 \%$, mais avec une différence moyenne $\bar{x}-\bar{y}$ de 0,099 en log, les nombres moyens d'UFC/ml ou de cellules/ $\mathrm{ml}$ ne sont pas significativement différents au seuil de $5 \%$.

L'écart type résiduel de la régression est significativement plus faible pour les laits de citernes au seuil de $5 \%$. Si $y$ est le nombre d'UFC/ml estimé à partir de la droite de calibrage, les limites de l'intervalle de confiance de $y$ (à $P=0,95$ ) sont en données non transformées, pour les laits d'entreprise, $y / 10^{0,823}$ soit $y / 6,656$ pour la limite inférieure, et $y \times 100,823$ soit $y$ $\times$ 6,656 pour la limite supérieure. Pour les échantillons de lait de producteurs, les limites de l'intervalle de confiance sont $y$ l 12,27 et $y \times 12,27$. Sur la totalité des 236 échantillons analysés, l'écart type résiduel de la régression est de 0,495 UFC/ml.

Dans le cas des échantillons prélevés dans les citernes de ramassage, la justesse du BactoFoss a pu être déterminée pour l'estimation de la flore totale après agitation standard ou à l'aide de l'Ultra-

Tableau II. Justesse du BactoFoss déterminée sur une population de 120 échantillons de lait de citernes pour l'estimation de 3 groupes microbiens. $s_{y, x}$ : écart type résiduel de la régression en $\log U \mathrm{FC} / \mathrm{ml}$; FTAS : flore totale après agitation standard; FTUT : flore totale après agitation Ultra-turrax; Psy : flore psychrotrophe.

Accuracy of BactoFoss determined with 120 dairy plant milk samples for the estimation of 3 microbial groups. $s_{y, x}$ : residual standard deviation of the regression in log CFU/ml; FTAS: total flora after standard shaking; FTUT : total flora after shaking with an Ultra-turrax; Psy : psychrotrophic flora.

\begin{tabular}{lcc}
\hline Flore & $s_{y, x}$ & Equation de régression \\
\hline FTAS & 0,420 & $Y=0,942 X+0,430$ \\
FTUT & 0,499 & $Y=0,924 X+0,844$ \\
Psy & 0,662 & $Y=1,067 X+0,008$ \\
\hline
\end{tabular}

turrax, ainsi que pour la flore psychrotrophe (tableau II). Dans les 3 cas, la pente et l'ordonnée à l'origine de la droite de calibrage ne sont pas significativement différentes, respectivement de 1,000 et de 0,000 au seuil de $5 \%$. Par contre, avec un écart type résiduel de $0,42 \log U F C / \mathrm{ml}$, la précision d'estimation du BactoFoss est significativement meilleure pour l'estimation de la flore totale après agitation standard.

\section{DISCUSSION}

Comme il existe très souvent un délai plus ou moins long entre le prélèvement des échantillons de lait et leur analyse microbiologique, ceux-ci sont habituellement conservés à $0^{\circ} \mathrm{C}$ dans la glace fondante, dans le but d'éviter toute multiplication microbienne au cours du stockage. Bien que le métabolisme cellulaire soit probablement affecté par l'abaissement de la température, nous n'avons pas mis en évidence, dans nos conditions d'analyse, d'effet significatif sur le dosage de l'ATP bactérien par le BactoFoss. En effet, l'écart type de reproductibilité au cours du temps du signal mesuré par le BactoFoss sur 2 échantillons de lait $\left(s_{R}=0,10\right.$ log cellules/ $\mathrm{ml}$ ) est très voisin de l'écart type de répétabilité de cet appareil calculé sur 236 échantillons de lait $\left(s_{R}=0,09\right.$ log cellules/ $\mathrm{ml}$ ). Ces observations sont en désaccord avec les travaux de Theron et al (1983), qui ont montré des effets de la température et du milieu de culture sur la quantité moyenne d'ATP par cellule. Cependant, ces auteurs ont travaillé avec des souches pures ensemencées sur milieu synthétique ou sur lait stérilisé et enrichi; dans ces conditions d'analyse, l'état physiologique des cellules est probablement très éloigné de celui rencontré dans du lait cru conservé à basse température.

Conformément à ce qui avait été montré par Olsen et Eriksen (1989) sur de la 
viande contaminée par 4 souches pures, la réponse du BactoFoss est linéaire sur une gamme de concentration de $10^{3}$ à $10^{7}$ UFC/ml. Cependant, nos essais de linéarité ont bien montré que le taux moyen d'ATP par cellule varie selon les échantillons analysés, entraînant des sousestimations ou des surestimations parfois importantes des nombres d'UFC/ml par le BactoFoss. Ces variations peuvent se justifier par des différences d'état physiologique ou de nature de la flore d'un échantillon de lait à l'autre. En effet, dans leurs essais de linéarité avec des souches pures, Olsen et Eriksen (1989) avaient obtenu sur de la viande, des droites parallèles et non confondues. De plus, des essais récents (Foss Electric, communication personnelle) ont également montré des variations, en fonction de la souche bactérienne, de l'efficacité d'extraction de I'ATP avec les réactifs actuellement utilisés, et ce particulièrement avec des souches de Pseudomonas.

Les nombres de bactéries déterminés par le BactoFoss à partir des dilutions des 2 échantillons atteignant des niveaux supérieurs à $10^{8} \mathrm{UFC} / \mathrm{ml}$, sont très sousestimés (environ 1 unité de logarithme). Deux raisons principales peuvent être invoquées. Tout d'abord, à ce niveau de concentration, les bactéries se trouvent en phase stationnaire, en raison de l'appauvrissement du milieu en éléments nutritifs et de l'accumulation de déchets toxiques. Cette phase se caractérise par un métabolisme microbien réduit, qui entraîne une diminution de la teneur en ATP par cellule. D'autre part, la chute du signal mesuré par le BactoFoss peut également être reliée à l'activité ATPasique des bactéries de l'espèce Pseudomonas fluorescens. En effet, Botha et al (1986) ont observé, en fin de croissance exponentielle et en début de phase stationnaire, une corrélation élevée ( $r=0,91$ ) entre le nombre de Pseudomonas fluorescens et l'activité ATPasique du milieu. II est donc possible que dans ces échantillons fortement contaminés, la quantité d'ATPase produite soit suffisante pour hydrolyser l'ATP extrait, et donc interférer sur son dosage.

Les variations de la teneur en ATP par cellule en fonction de la nature et/ou de l'état physiologique des bactéries sont sans doute également en partie à l'origine de la différence observée entre les précisions d'estimation du BactoFoss obtenues pour les laits de producteurs et de citernes. En effet, les laits de citernes ont en général fait l'objet d'une conservation prolongée à basse température, entraînant une prolifération exclusive des Pseudomonas psychrotrophes (Richard, 1981). Le fait d'avoir obtenu une meilleure relation entre Référence et BactoFoss pour les laits de citernes peut ainsi résulter de 2 facteurs : une flore relativement homogène et des bactéries en phase de croissance. À l'inverse, les échantillons de lait de producteurs proviennent en général d'un mélange d'une ou 2 traites, qui a donc fait l'objet d'une conservation limitée; la nature de la flore et son métabolisme varient de façon plus importante d'un échantillon à l'autre.

Avec un écart type résiduel de 0,420 log UFC/ml pour les laits de citernes, et de $0,556 \mathrm{log} U \mathrm{FC} / \mathrm{ml}$ pour les laits de producteurs, les résultats de notre étude sont très éloignés de ceux obtenus par Eriksen et Olsen (1988) sur un prototype $\left(s_{y, x}=0,27\right.$ $\log \mathrm{UFC} / \mathrm{ml}$ ). Cependant, la comparaison entre les 2 études est délicate, compte tenu des différences existant au niveau de la technique de référence et du protocole opératoire de dosage de l'ATP : préchauffage ou non des échantillons de lait $\left(40^{\circ} \mathrm{C}\right)$, mode d'homogénéisation du lait avant passage au BactoFoss (homogénéisateur ou manuelle), expression du signal mesuré par le BactoFoss (unités lumineuses arbitraires ou cellules/ml). 
De plus, le BactoFoss possède une précision d'estimation nettement moins satisfaisante, qu'une technique manuelle, comprenant également une étape de filtration : I'ATP-F test, proposé par Lumac. En effet, sur une population de 92 échantillons de lait de citernes couvrant une gamme de concentration de $10^{4}$ à $10^{7} \mathrm{UFC} / \mathrm{ml}$, Rongvaux-Gaïda et Piton (1991) ont obtenu un écart type résiduel $s_{y, x}$ de $0,30 \mathrm{log}$ $\mathrm{UFC} / \mathrm{ml}$. De même, sur 285 échantillons couvrant une gamme de concentration similaire, Van Crombrugge et al (1989) ont obtenu un $s_{y, x}$ de $0,27 \mathrm{log}$ UFC/ml. Bien que reposant sur le même principe, le BactoFoss et I'ATP-F test présentent de nombreuses différences de mise en œuvre. Le BactoFoss peut en effet être défini comme une microtechnique (volume d'échantillon de $70 \mu \mathrm{l}$ contre $5 \mathrm{ml}$ ) qui utilise un temps d'extraction et de digestion de I'ATP somatique beaucoup plus court que I'ATP-F test; de plus, les réactifs et les membranes de filtration sont totalement différents. Par contre, avec un GRSD de $28 \%$ (exprimé en $\%$ UFC/ml), le BactoFoss possède une répétabilité plus satisfaisante que I'ATP-F test (GRSD $=43 \%$ d'après RongvauxGaïda et Piton, 1991). L'automatisation est certainement à l'origine de cette amélioration de la répétabilité, car elle diminue les erreurs aléatoires liées au facteur humain (pipettage, temps de contact, etc).

En définitive, le BactoFoss présente de nombreux avantages liés à son automatisation, sa facilité d'utilisation, sa rapidité de réponse et enfin, certaines de ses caractéristiques analytiques comme la limite de détermination et la fidélité. Cependant, il ne permet pas l'évaluation de la qualité bactériologique du lait cru avec la justesse annoncée par Eriksen et Olsen (1988). Avec un écart type résiduel de 0,42 log UFC/ml pour les laits de citernes à l'arrivée au quai, le BactoFoss présente une précision d'estimation assez éloignée de celle de techniques directes comme la DEFT $\left(s_{y, x}=0,25 \log\right.$ UFC/ml, d'après Dasen et al, 1987) ou de techniques indirectes comme I'ATP-F test ou la microrespirométrie $\left(s_{y, x}=0,18 \mathrm{log} \mathrm{UFC} / \mathrm{ml}\right.$, d'après Rongvaux-Gaïda et al, 1990). À l'inverse, la précision d'estimation du BactoFoss est tout à fait comparable à celle d'une technique de dosage d'une activité microbienne comme la conductimétrie (Piton et Dasen, 1988; Piton et Rongvaux-Gaïda, 1990).

II est désormais bien connu que le mode d'agitation des échantillons de lait cru joue un rôle très important sur les résultats de dénombrement de sa flore (Huhtanen et al, 1970; Te Whaiti et Fryer, 1977; Richard, 1980; Richard et al, 1981; Piton et Richard, 1983). Jusqu'à présent, c'est probablement l'agitation par turbine (Ultra-turrax) qui assure la meilleure dispersion des amas microbiens (Richard, 1980; Richard et al, 1981). II est donc très intéressant de constater que l'homogénéisateur à billes proposé par Foss Electric possède une efficacité semblable à la turbine, et ce à tous les niveaux de pollution micro-bienne du lait. II présente en effet certains avantages par rapport à l'Ultraturrax, comme la rapidité, la simplicité et le confort sonore d'utilisation. Quelques précautions d'emploi sont cependant nécessaires, en particulier au niveau de la désinfection de l'appareil et de l'agitation des échantillons avant leur passage à l'homogénéisateur. En effet, cet appareil n'utilise qu'une fraction de l'échantillon (environ $25 \mathrm{ml}$ ); ainsi, il est évident qu'il ne peut donner un résultat précis que si l'échantillon a subi auparavant une agitation correcte, par exemple une agitation standard (Anonyme, 1978) dans un flacon rempli aux $3 / 4$. Un protocole précis d'utilisation de I'homogénéisateur doit donc être fourni avec l'appareil.

Compte tenu de l'efficacité de dispersion des amas microbiens par l'homogé- 
néisateur, il est surprenant de constater que la précision d'estimation du BactoFoss est meilleure lorsqu'on compare ses résultats à ceux de dénombrement obtenus après agitation standard plutôt qu'après passage à l'Ultra-turrax. Ce fait peut être attribué à un artefact statistique, car l'écart type résiduel des écarts à la régression dépend de la variance de la variable expliquée. Or, les résultats de numération ont une variance $\left(s^{2}\right)$ plus élevée après une agitation violente, soit $s=0,290$ contre $s=$ $0,209 \mathrm{log}$ UFC/ml pour l'agitation standard.

Enfin, conformément à ce qui avait déjà été montré par Piton et Richard (1983), l'effet du mode d'agitation des échantillons sur les résultats de dénombrement de la flore totale semble varier en fonction du niveau de pollution microbienne. Cela peut s'expliquer par les changements de nature de flore en fonction des niveaux de contamination. En effet, les échantillons peu contaminés contiennent en général, en flore dominante, des microcoques et des streptocoques; par contre, la proportion de bactéries à Gram négatif devient très importante dans les laits très pollués (Thomas et al, 1962; Twomey et Crawley, 1968; Richard, 1981 et 1983). Or Te Whaiti et Fryer (1977) ont montré que dans le lait cru conservé à basse température, les bactéries psychrotrophes à Gram négatif se trouvent sous forme d'amas, dont l'importance augmente avec la durée de stockage à $4^{\circ} \mathrm{C}$, mais d'après Richard (1980) les amas formés par les bâtonnets à Gram négatif sont beaucoup plus faciles à dissocier que ceux formés par les microcoques et les streptocoques.

\section{REMERCIEMENTS}

Les auteurs remercient la société Foss Electric France (35, rue des Peupliers, 92000 Nanterre) pour le prêt de BactoFoss, la fourniture des réactifs nécessaires au test, ainsi que pour son assistance technique.
Les auteurs tiennent également à remercier les entreprises laitières suivantes, pour le prélèvement et l'envoi des échantillons de lait : la société Bel, centre de recherches, 21 , avenue de Ronsard, 41100 Vendôme; la société GervaisDanone (BSN) par l'intermédiaire de la société Stenval, 5, route de Blanche, 59270 Bailleul; la société Yoplait : l'usine d'Amiens (80000 Sully) et l'usine de Ressons/Matz (60490 Elnor). Ce travail a reçu le soutien financier de la Direction générale de l'alimentation du ministère de l'Agriculture (Contrat DGAL $n^{\circ} \mathrm{R} 88 / 11$ ).

\section{RÉFÉRENCES}

Anonyme (1978) Standard methods for the examination of dairy products. 14th edn, American Public Health Association, New York

Anonyme (1985a) Milk. Definition and evaluation of the overall accuracy of indirect methods of milk analysis. Application to calibration procedure and quality control in dairy laboratory. Norme provisoire FIL-IDF 128

Anonyme (1985b) Standard methods for the examination of dairy products. 15th edn, American Public Health Association, New York

Anonyme (1987) Microbiologie alimentaire. Ensemencement et dénombrement des microorganismes à l'aide du système "Spiral». Norme française NF V 08-100 AFNOR, Paris

Bossuyt R (1978) Usefulness of an ATP assay technique in evaluation of the somatic cell content of milk. Milchwissenschaft 33, 11-13

Bossuyt R (1982) A 5-minute ATP platform test for judging the bacteriological quality of raw milk. Neth Milk Dairy J 36, 355-364

Botha WC, Lück H, Jooste PJ (1986) Determination of bacterial ATP in milk. The influence of adenosine triphosphate-hydrolyzing enzymes from somatic cells and Pseudomonas fluorescens. J Food Prot 49, 822-825

Dasen A, Piton C, Grappin R, Guerry P (1987) Évaluation de la technique DEFT associée à un comptage visuel ou à un comptage par analyseur d'images pour la numération de la flore totale du lait cru. Lait 67, 77-95

Eriksen B, Olsen O (1988) Rapid assessment of the microbial status of bulk milk and raw meat with the new instrument: BactoFoss. Presented at ATP 88 , Symposium on rapid 
microbiology, university of Sussex, Brighton, England, April 14th-15th

Gilchrist JE, Campbell JE, Donnelly CB, Peeler JT, Delaney JM (1973) Spiral plate method for bacterial determination. Appl Microbiol 25, 244-252

Huhtanen CN, Brazis AR, Arledge WL, Cook EW, Donnelly CB, Ginn RE, Murphy JN, Randolph HE, Sing EL, Thompson DI (1970) Effect of dilution bottle mixing methods on plate counts of raw milk bacteria. I Milk Food Technol 33, 269-273

Langeveld LPM, Van der Waals CB (1988) The ATP platform test, the $\triangle$ ATP test and the direct microscopic count procedure as methods of estimating the microbial quality of raw milk. Neth Milk Dairy J 42, 173-182

Olsen O, Eriksen B (1989) Introduction of BactoFoss. A newly developed instrument for assessment of the microbial status of raw meat. In: Rapid methods and automation in microbiology and immunology. 5th Int Symposium, Florence, 1987 (Balows A, Tilton RC, Turano A, eds) Brixia Academic Press, Brescia

Piton C, Richard J (1983) Influence de l'agitation des échantillons de lait cru sur les résultats de dénombrement de trois groupes microbiens d'intérêt technologique. Lait 63, 405415

Piton C, Dasen A (1988) Évaluation de la mesure d'impédance comme technique rapide d'appréciation de la qualité bactériologique du lait cru. Lait 68, 467-484

Piton C, Rongvaux-Gaïda D (1990) Estimation par conductimétrie de la flore aérobie mésophile, des bactéries coliformes et de la flore psychrotrophe du lait cru. Lait 70, 293-306

Piton C, Grappin R (1991) A model for statistical evaluation of precision parameters of microbiological methods: application to dry rehydratable film methods and the IDF reference methods for enumeration of total aerobic mesophilic flora and coliforms in raw milk. $J$ Assoc Off Anal Chem 74, 72-103

Richard J (1980) Influence de l'agitation du lait cru sur les résultats de dénombrement de sa flore totale à l'aide d'une anse calibrée. Lait 60, 211-225

Richard J (1981) Influence de diverses méthodes de nettoyage des machines à traire sur la "qualité de conservation" du lait cru à basse température. Lait 61, 354-369

Richard J (1983) Nature de la flore microbienne dominante et sous-dominante des laits crus très pollués. Lait $63,148-170$

Richard J, Krebs C, Walter J, Grappin R (1981) Comparaison de divers modes d'agitation des échantillons de lait cru en vue de la numération de sa flore totale suivant la méthode Thompson. Lait 61, 250-260

Rongvaux-Gaîda D, Piton C (1991) Comparaison de trois méthodes de dosage de l'ATP par bioluminescence pour l'estimation de la qualité bactériologique du lait cru. Lait, 483491

Rongvaux-Gaïda D, Peroz A, Verdier B, Piton C (1990) Estimation de la qualité bactériologique du lait cru par microrespirométrie. Lait 70, 23-36

Te Whaiti IE, Fryer TF (1977) The enumeration of bacteria in refrigerated milk. $N Z J$ Dairy Sci Technol 12, 51-57

Theron DP, Prior BA, Lategan PM (1983) Effect of temperature and media on adenosine triphosphate cell content in Enterobacter aerogenes. J Food Prot 46, 196-198

Theron DP, Prior BA, Lategan PM (1986) Determination of bacterial ATP levels in raw milk: selectivity of non-bacterial ATP hydrolysis. $J$ Food Prot 49, 4-7

Thomas SB, Hobson PM, Bird ER, King KP, Druce RG, Cox DR (1962) The microflora of raw milk as determined by plating on Yeastrel Milk Agar incubated at $30^{\circ} \mathrm{C}$. J Appl Bacteriol 25, 107-115

Twomey A, Crawley WE (1968) The microflora of raw milk. NZJ Dairy Sci Technol 3, 44-48

Van Crombrugge JV, Waes G, Reybroeck W (1989) The ATP-F test for estimation of bacteriological quality of raw milk. Neth Milk Dairy J 43, 347-354

Waes G, Van Crombrugge J, Reybroeck W (1989) The ATP-F test for estimation of the bacteriological quality of raw milk. In: Proceedings of international seminar on modern microbiological methods for dairy products, Santander, May. IDF Spec Issue 8901, 279287 